\title{
Caracterização de mulheres hospitalizadas por neoplasia maligna da mama na Bahia, Brasil, 2012-2016
}

\author{
Characterization of woman due to breast cancer \\ in Bahia, Brazil, 2012 -2016
}

\author{
Beatriz Emanuele dos Santos e Matos ${ }^{1}$ (1) \\ Nathalia Alves Mendes Pereira ${ }^{2}$ (1) \\ Flavia Carvalho Rocha 3 (1) \\ Cristina Aires Brasil ${ }^{4}$ (1) \\ Augusto Cesar Costa Cardoso 5 \\ Catia Suely Palmeira 6 (1)
}

\begin{abstract}
1'Autora para correspondência. Escola Bahiana de Medicina e Saúde Pública (Salvador). Bahia, Brasil. beatrizemanuele1999@gmail.com 2-6Escola Bahiana de Medicina e Saúde Pública (Salvador). Bahia, Brasil. nathaliaamp@icloud.com, faaucarvalho1@gmail.com, cristinabrasil@bahiana.edu.br, acccardoso@uneb.br, catia_palmeira@yahoo.com.br
\end{abstract}

RESUMO | OBJETIVO: Caracterizar as mulheres hospitalizadas por neoplasia maligna da mama na Bahia no período de 2012 a 2016. MÉTODo: Estudo descritivo, ecológico de serie temporal, utilizando dados secundários do Sistema de Informação hospitalar SIH (SUS) no período de 2012 a 2016 no estado da Bahia, disponíveis no site do DATASUS. A população foi constituída de mulheres na faixa etária entre 20 a 59. As seguintes variáveis foram analisadas: faixa etária, macrorregião de ocorrência, raça/cor. RESULTADOS: No período ocorreram 11.787 internações por neoplasia maligna da mama, maior proporção em mulheres com idade de 50 a 59 anos $(42,6 \%)$ e de cor/raça parda. A macrorregião do Leste do Estado apresentou maior frequência com $(57,1 \%)$ de internações. A taxa de mortalidade entre as mulheres internadas foi maior na faixa etária de 50 a 59 anos (9,7\%). CONCLUSÃO: Mulheres com idade de 50 a 59 anos e de cor/ raça parda são as que mais se internaram por câncer de mama. Os dados apresentados permitem traçar um perfil da morbidade por câncer na Bahia e suas macrorregiões e servir de subsídios para estratégias de enfrentamento do problema.

DESCRITORES: Câncer da mama. Mulheres. Hospitalização. Sistemas de Informação em Saúde.

\begin{abstract}
OBJECTIVE: To characterize women hospitalized for malignant breast cancer in Bahia from 2012 to 2016. METHOD: Descriptive study, ecologic of time serie, using secondary data were gathered from the Hospital Information System (SIH-SUS) on period of 2012 to 2016 on State of Bahia, available on site of DATASUS. The population consisted of women in the age group between 20 and 59 years. The following variables were used: age group, macro region of incident, race/color. RESULTS: In the period, there were 11.787 hospitalizations for breast cancer with larger proportion in women on age of 50 to 59 years old $(42,6 \%)$ and color/race brown. The macro region of East of State presented larger frequency $(57,1 \%)$ of hospital admissions. The death rate between women hospitalized was bigger in age category of 50 to 59 years old $(9,7 \%)$. CONCLUSION: Women on age 50 to 59 years old and color/race brown are the most hospitalized due to breast cancer. The data submitted allowed chart a profile of morbidity for cancer in Bahia and yours macro regions and served of subsidies for strategy of trouble confrontation.
\end{abstract}

KEYWORD: Breast Neoplasms. Women. Hospitalization. Health Information System. 


\section{Introdução}

Atualmente o câncer de mama representa importante problema de saúde pública em todo o mundo, sendo a neoplasia maligna com maior incidência e a principal causa de morte por câncer em mulheres, tanto nos países em desenvolvimento quanto nos desenvolvidos ${ }^{1}$. As últimas estatísticas mundiais do Globocan $2018^{1}$ estimaram 2,1 milhões de casos novos de câncer e 627 mil óbitos pela doença. No Brasil, no ano de 2016 o câncer de mama foi responsável por 16.069 mortes de mulheres no país e as estimativas de incidência para o ano de 2019 foram de 59.700 casos novos, representando $29,5 \%$ dos cânceres em mulheres².

A neoplasia maligna da mama tem grande impacto para a sociedade e serviços de saúde, pois ocasionam custos associados à morte prematura e à perda de produtividade causada pelo aumento expressivo nos afastamentos temporários e definitivos ${ }^{3}$.

No Brasil, dentre todas as neoplasias, a maligna de mama representa $19 \%$ das causas de aposentadorias por invalidez. Além disso, é considerada importante causa de internação devido à complexidade crescente do tratamento, na maioria das vezes, representado pelo procedimento cirúrgico e tratamento quimioterápico. As possíveis complicações do paciente com câncer, muitas das vezes, requerem também uma assistência hospitalar para a reabilitação do seu quadro clínico ${ }^{4}$.

O câncer de mama é uma doença resultante da multiplicação de células anormais da mama, formando um tumor, sendo que alguns tipos se desenvolvem rapidamente e outros não. Os principais fatores de riscos para o desenvolvimento da neoplasia maligna da mama estão relacionados com história familiar e pessoal, fatores endócrinos e genéticos, idade, características reprodutivas, como a menarca precoce e menopausa tardia, e ainda com os hábitos de vida e as influências ambientais 5 .
As novas diretrizes para detecção precoce de neoplasia maligna da mama no Brasil, destinadas respectivamente a mulheres assintomáticas e casos com sinais e sintomas sugestivos de câncer, são divididas em recomendações para rastreamento e para diagnóstico precoce ${ }^{6}$.

A situação da detecção precoce do câncer de mama por meio da mamografia e o acesso ao diagnóstico e ao tratamento no Brasil ainda é crítica. A cobertura da mamografia no país é baixa, comparada à preconizada pela Organização Mundial da Saúde (OMS) e com diferenças importantes tanto na distribuição da oferta e utilização dos procedimentos de detecção precoce do câncer de mama, quanto na cobertura e no desequilíbrio entre o que é produzido e a necessidade social estimada7. Os autores sinalizam o descompasso entre o estímulo à realização da mamografia e a estrutura correspondente para investigação diagnóstica na rede de serviços do SUS.

Atualmente as modalidades terapêuticas para o câncer de mama são indicadas de acordo com aspectos biológicos e determinadas características específicas de cada usuária, como idade, presença ou não de comorbidades, preferências e, sobretudo considerando o estadiamento do tumor ${ }^{2}$.

Informações sobre incidência, morbidade hospitalar e mortalidade são medidas que permitem analisar a ocorrência, a distribuição e a evolução das doenças. Apesar das causas das internações hospitalares no Brasil constituírem um campo de interesse de pesquisa na área de saúde, existe uma lacuna de estudo com dados referentes ao câncer de mama na Bahia.

Assim, conhecer informações sobre o perfil das internações de determinado tipo de câncer na Bahia mediante registros de morbidade e mortalidade provenientes do Sistema de Informações fornecem indicadores locais que podem nortear estratégicas de planejamento de programas de prevenção e de controle do problema de saúde em questão.

Deste modo, esse estudo tem como objetivo caracterizar as mulheres hospitalizadas por neoplasia maligna da mama na Bahia, no período de 2012 a 2016. 


\section{Métodos}

Trata-se de um estudo descritivo, ecológico de serie temporal. Foram utilizados dados secundários obtidos no Sistema de Informação Hospitalares SIH (SUS) disponibilizados pelo Ministério da Saúde, no site www.datasus.gov.br, informações de saúde/TABNET/ epidemiológicas e morbidade no grupo Morbidade Hospitalar do SUS.

O SIH é o sistema de mapeamento de internação em unidades hospitalares participantes do SUS (públicas ou particulares conveniadas), sendo que as internações custeadas diretamente ou cobertas por segurosaúde não são contadas. Para coleta, foi selecionado o item contido na lista de morbidade da Classificação Internacional de Doenças CID-10, C50 - representado pelo termo neoplasia maligna da mama.

Os dados coletados se referem ao período de 2012 a 2016 e foram acessados em novembro de 2018. Foram incluídas somente mulheres na faixa etária entre 20 a 59. As variáveis selecionadas para análise foram: faixa etária, macrorregião de ocorrência, raça/cor. Excluiu-se da análise os casos registrados no DATASUS como "sem informações" para qualquer uma das variáveis selecionadas.
A análise dos dados deu-se a partir da frequência absoluta dos casos de internações de mulheres com neoplasia maligna da mama pelas variáveis consideradas e cálculo da proporção. A taxa de mortalidade é representada pela razão entre a quantidade de óbitos e o número de Autorização de internação hospitalar - AlH aprovadas, computadas como internações, no período, multiplicada por 100. Para o cálculo dos indicadores e elaboração das tabelas, foram utilizados os softwares Tabnet e o Excell 2010.

O estudo seguiu as normas dispostas na Resolução 466/12 do Conselho Nacional de Ética em Pesquisa e por utilizar dados secundários de domínio público, dispensa autorização do comitê de ética em pesquisa.

\section{Resultados}

Nos anos de 2012 a 2016, no Estado da Bahia, foi registrado um total de 11.787 internações de mulheres com idade entre 20 a 59 anos por neoplasia maligna da mama. O maior registro foi observado no ano de 2016 (2.543 casos de internações). A maior frequência de internações foi observada entre as mulheres com idade entre 50 aos 59 anos (42,6\%), seguida das mulheres com 40 e 49 anos (38\%) conforme pode ser observado na Tabela 1.

Tabela 1. Internações de mulheres por neoplasia maligna de mama segundo faixa etária. Bahia, 2012 a 2016

\begin{tabular}{|c|c|c|c|c|c|c|c|c|c|c|}
\hline \multirow{2}{*}{ Faixa Etária } & \multicolumn{2}{|c|}{2012} & \multicolumn{2}{|c|}{2013} & \multicolumn{2}{|c|}{2014} & \multicolumn{2}{|c|}{2015} & \multicolumn{2}{|c|}{2016} \\
\hline & $\mathbf{N}^{\circ}$ & $\%$ & $\mathbf{N}^{\circ}$ & $\%$ & $\mathrm{~N}^{\circ}$ & $\%$ & $\mathbf{N}^{\circ}$ & $\%$ & $\mathbf{N}^{\circ}$ & $\%$ \\
\hline 20 a 29 anos & 67 & 2,9 & 111 & 4,7 & 85 & 4,0 & 109 & 4,4 & 86 & 3,4 \\
\hline 30 a 39 anos & 375 & 16,3 & 377 & 15,9 & 361 & 17,2 & 427 & 17,3 & 407 & 16,0 \\
\hline 40 a 49 anos & 946 & 41,0 & 939 & 39,7 & 826 & 39,3 & 969 & 39,2 & 967 & 38,0 \\
\hline 50 a 59 anos & 919 & 39,8 & 938 & 39,7 & 829 & 39,5 & 966 & 39,1 & 1083 & 42,6 \\
\hline Total & 2307 & 100,0 & 2365 & 100,0 & 2101 & 100,0 & 2471 & 100,0 & 2543 & 100,0 \\
\hline
\end{tabular}

Fonte: Ministério da Saúde - Sistema de Informações Hospitalares do SUS (SIH/SUS)- 01/11/2018 
No que se refere a cor/raça, os percentuais de internação foram acentuadamente maiores entre as mulheres que se autodeclaram de cor parda, em todos os anos observados (Tabela 2).

Tabela 2. Internações de mulheres por neoplasia maligna de mama segundo cor/raça. Bahia, 2012 a 2016

\begin{tabular}{|c|c|c|c|c|c|c|c|c|c|c|}
\hline \multirow{2}{*}{ Cor/raça } & \multicolumn{2}{|c|}{2012} & \multicolumn{2}{|c|}{2013} & \multicolumn{2}{|c|}{2014} & \multicolumn{2}{|c|}{2015} & \multicolumn{2}{|c|}{2016} \\
\hline & $\mathbf{N}^{\circ}$ & $\%$ & $\mathbf{N}^{\circ}$ & $\%$ & $\mathbf{N}^{0}$ & $\%$ & $\mathbf{N}^{\circ}$ & $\%$ & $\mathbf{N}^{\circ}$ & $\%$ \\
\hline Branca & 234 & 7,3 & 323 & 9,5 & 249 & 8,2 & 309 & 8,6 & 302 & 8,4 \\
\hline Preta & 207 & 6,5 & 419 & 12,3 & 357 & 11,8 & 428 & 11,9 & 403 & 11,2 \\
\hline Parda & 1353 & 42,5 & 2099 & 61,4 & 2154 & 71,1 & 2587 & 71,9 & 2625 & 72,8 \\
\hline Amarela & 3 & 0,1 & 9 & 0,3 & 8 & 0,3 & 17 & 0,5 & 25 & 0,7 \\
\hline Total & 3185 & 100,0 & 3417 & 100,0 & 3030 & 100,0 & 3596 & 100,0 & 3605 & 100,0 \\
\hline
\end{tabular}

Fonte: Ministério da Saúde - Sistema de Informações Hospitalares do SUS (SIH/SUS)- 01/11/2018

Na tabela 3, a análise da ocorrência de mulheres internadas por neoplasia maligna da mama, segundo as macrorregiões, revela que a macrorregiões Leste, Centro leste e Sul, ocupam as três primeiras posições em todo o período analisado. A região Leste mostrou ocorrência de internações em 2012 (57,1\%),a região no Centro Leste em 2013 (15,4\%) e a Sul em 2012 e 2014 com (10\%). As demais regiões não chegaram a ultrapassar a média de $7 \%$ das ocorrências.

Tabela 3. Internações de mulheres por neoplasia maligna da mama segundo Macrorregião de Saúde. Bahia, 2012 a 2016

\begin{tabular}{|c|c|c|c|c|c|c|c|c|c|c|}
\hline \multirow{2}{*}{ Macrorregião de Saúde } & \multicolumn{2}{|c|}{2012} & \multicolumn{2}{|c|}{2013} & \multicolumn{2}{|c|}{2014} & \multicolumn{2}{|c|}{2015} & \multicolumn{2}{|c|}{2016} \\
\hline & $\mathrm{N}^{\circ}$ & $\%$ & $\mathrm{~N}^{\mathrm{o}}$ & $\%$ & $\mathrm{~N}^{\circ}$ & $\%$ & $\mathrm{~N}^{\mathrm{O}}$ & $\%$ & $\mathrm{~N}^{\circ}$ & $\%$ \\
\hline Centro-Leste & 348 & 10,9 & 527 & 15,4 & 449 & 14,8 & 408 & 11,3 & 425 & 11,8 \\
\hline Centro-Norte & 111 & 3,5 & 163 & 4,8 & 89 & 2,9 & 119 & 3,3 & 132 & 3,7 \\
\hline Extremo Sul & 54 & 1,7 & 86 & 2,5 & 108 & 3,6 & 147 & 4,1 & 164 & 4,5 \\
\hline Leste & 1820 & 57,1 & 1763 & 51,6 & 1548 & 51,1 & 1944 & 54,1 & 1928 & 53,5 \\
\hline Nordeste & 126 & 4,0 & 169 & 4,9 & 124 & 4,1 & 166 & 4,6 & 135 & 3,7 \\
\hline Norte & 177 & 5,6 & 226 & 6,6 & 184 & 6,1 & 170 & 4,7 & 235 & 6,5 \\
\hline Oeste & 66 & 2,1 & 50 & 1,5 & 61 & 2,0 & 84 & 2,3 & 109 & 3,0 \\
\hline Sudoeste & 164 & 5,1 & 149 & 4,4 & 164 & 5,4 & 225 & 6,3 & 213 & 5,9 \\
\hline Sul & 319 & 10,0 & 284 & 8,3 & 303 & 10,0 & 333 & 9,3 & 264 & 7,3 \\
\hline Total & 3185 & 100,0 & 3417 & 100,0 & 3030 & 100,0 & 3596 & 100,0 & 3605 & 100,0 \\
\hline
\end{tabular}

Fonte: Ministério da Saúde - Sistema de Informações Hospitalares do SUS (SIH/SUS)- 01/11/2018 
A maior taxa de mortalidade de mulheres por neoplasia maligna da mama ocorreu entre as mulheres de 50-59 anos em 2012 e 2015 (9,7 e 9,2, respectivamente). Observa-se que no ano de 2016 a taxa de mortalidade mostra-se reduzida em relação aos anos anteriores. As faixas etárias 30 a 39 anos e 40 a 49 anos também se destacaram pelo elevado número de taxa de mortalidade no ano de $2013(8,5)$ e no ano de $2014(8,2)$ respectivamente $(T a b e l a 4)$.

Tabela 4. Taxa de mortalidade de mulheres por neoplasia maligna da mama segundo faixa etária. Bahia,2012 a 2016

\begin{tabular}{lccccc}
\hline \multirow{2}{*}{ Faixa etária } & \multicolumn{5}{c}{ Taxa de mortalidade por $\mathbf{1 0 0}$ casos de internação } \\
\cline { 2 - 6 } 20 a 29 anos & 2012 & 2013 & 2014 & 2015 & 2016 \\
\hline 0 a 39 anos & 1,5 & 2,7 & 3,5 & 6,4 & 3,5 \\
40 a 49 anos & 6,4 & 8,5 & 6,9 & 5,9 & 6,9 \\
50 a 59 anos & 7,4 & 6,3 & 8,2 & 5,1 & 7,2 \\
\hline
\end{tabular}

Fonte: Ministério da Saúde - Sistema de Informações Hospitalares do SUS (SIH/SUS)- 01/11/2018

\section{Discussão}

No presente artigo o número de internações foi maior entre mulheres na faixa etária de 50 a 59 anos. Esse resultado pode ser explicado parcialmente pela relação das características individuais como a idade, que se constitui um dos mais importantes fatores de risco para o desenvolvimento e prognóstico da neoplasia maligna da mama. O câncer de mama é raro antes dos 30 anos de idade, tem aumento da sua incidência até os 50 anos e, posteriormente, ocorre de forma mais lenta. No entanto, nos últimos anos tem sido observada em todo o mundo, uma elevação da incidência dessa neoplasia, inclusive em faixas etárias mais jovens ${ }^{5}$.

Considerando o acompanhamento em serviços públicos como uma variável proxy da posição socioeconômica individual compatível com menor poder aquisitivo, e que no Brasil pardos e pretos encontraram-se em desvantagem socioeconômica quando comparados aos brancos, talvez estas condições poderiam explicar a maior taxa internação por câncer de mama em mulheres que se autodeclaram parda.
Ainda que cor/raça não se constitua em uma categoria adequada para definir biologicamente a espécie humana, como conceito social tem a sua importância, por estar associada às iniquidades de saúde 9 . Neste sentido, como a composição étnica de cada local pode estar atrelada às possibilidades de acesso ao diagnóstico e ao tratamento ${ }^{10}$, e na Bahia pardos e pretos, compõem os extratos sociais menos favorecidos, são predominantes, é esperado que utilizem mais os serviços hospitalares públicos de saúde. No estudo de Medeiros et al. ${ }^{10}$, realizado no Brasil, foi observado que mulheres pretas, pardas, indígenas e amarelas apresentaram maiores chances de receberem tratamento tardio que as mulheres de cor/raça da pele branca. Em um país com as dimensões do Brasil, a distribuição desigual da população quanto ao acesso aos serviços de saúde marca um desafio a ser enfrentado.

As macrorregiões analisadas mostraram que a região leste da Bahia está em primeiro lugar na ocorrência de mulheres internadas com neoplasia maligna da mama. Este resultado pode ser justificado, por a macrorregião leste apresentar cidades mais populosas como a capital Salvador, e consequentemente, mais oferta de serviços hospitalares adequados à realização da mastectomia. 
Pode-se inferir, ainda, que muitas mulheres por realizarem a internação na capital se hospedaram neste local e fornecem esse endereço como de sua residência. Estudo sobre tendência da mortalidade por câncer nas capitais e interior do Brasil entre 1980 e 2006, mostrou que o aumento da mortalidade por câncer de mama apresentou taxas sempre mais altas nas capitais ${ }^{11}$. Além disso, nascer em uma região diferente daquela da instituição de tratamento está associada a uma chance $12 \%$ maior de estar em estágio avançado da doença do que no estágio inicial ${ }^{12}$. Os autores afirmam que o acesso ao diagnóstico de câncer de mama no Brasil é desigual e que fatores socioeconômicos podem influenciar o estágio do diagnóstico. Com isso, os autores destacam a necessidade de promover um acesso mais amplo ao diagnóstico do câncer no Brasil com especial atenção às mulheres com menor nível socioeconômico.

Diferente de diversos países desenvolvidos, o Brasil tem registrado nos últimos anos aumento da taxa de mortalidade por câncer de mama, justificado, sobretudo, pelo diagnóstico tardio e pelo atraso na implantação do tratamento adequado, propiciando o crescimento tumoral, tornando o prognóstico da população vulnerável reservado, apesar de que essa neoplasia é considerada curável se diagnosticada e tratada precocemente ${ }^{13}$.

Na assistência oncológica à pessoas acometidas por neoplasia mamária, o tempo de atraso pode compreender desde os primeiros sintomas até a primeira consulta, passando acesso ao serviço de referência especializado, até a realização do tratamento específico. Considerando que esse período de tempo é crucial para a cura e recuperação do paciente, o Ministério da Saúde do Brasil determinou, por meio de portaria, que o tempo entre o registro do diagnóstico de câncer no prontuário médico e o início do tratamento não deve ultrapassar 60 dias $^{14}$.

O estudo de Renna et al. ${ }^{12}$, cujo objetivo foi analisar os fatores relacionados ao diagnostico do câncer de mama em estagio avançado no Brasil entre 2000 e 2012, observou que o tempo desde a data do diagnóstico até o início do tratamento variou de 0 a 182 dia e em $36,2 \%$ dos casos, o tempo entre o diagnóstico e o início do tratamento do câncer ultrapassou 60 dias.
Diferenças na sobrevida pela doença ocorrem em regiões que apresentavam ho-mogeneidade de atenção à saúde com relação aos protocolos clínicos de abordagem em oncologia, o que pode representar diagnóstico tardio ou classificação inadequada do tumor, ocasionando tratamento não realizado tardio ou inadequado. Geralmente mulheres com câncer de mama que não dispõe de seguro de saúde privado são diagnosticadas mais tardiamente e morrem mais precocemente, quando comparadas com mulheres com a doença, mas que tem o referido seguro ${ }^{9}$.

A maioria das mulheres diagnosticadas com câncer de mama em países de alta renda pode razoavelmente esperar ser curada e ter uma expectativa de vida mais longa, em função dos serviços de saúde ser mais avançados na triagem e no tratamento ${ }^{3}$. Para os autores para reduzir a mortalidade por câncer, é necessário reduzir incidência de câncer e melhorar a sobrevivência do câncer.

Estudo aponta que grande número de mortes evitáveis se deve em grande parte ao acesso diferenciado ao tratamento do câncer entre os grupos com maior poder aquisitivo e carentes, e que muitas das mortes seriam evitáveis, principalmente se serviços de boa qualidade fossem acessíveis a toda a população feminina ${ }^{15}$.

Constatou-se no presente estudo que a maior taxa de mortalidade foi na faixa etária 50 a 59 anos. A mortalidade apresenta associação positiva/significativa com a longevidade em todos os tipos de câncer incluindo o da mama².

Entre algumas das explicações dadas a este fato é que as pessoas mais velhas, cem certa frequência, tendem a diminuir a realização dos exames de rotina necessários, tornando-se mais vulneráveis a descoberta de tumores em estágios mais avançados, e consequentemente a tratamentos cirúrgicos mais radicais, o que reduz a chance de cura, aumenta as possibilidades de complicações e a permanência hospitalar ${ }^{16}$. 
A literatura mostra que taxa de mortalidade por câncer de mama está associada de forma negativa com o nível de gastos públicos em saúde e varia conforme a região, sendo que essa heterogeneidade regional pode ser um reflexo de características geográficas, demográficas, socioeconômicas e culturais. No Brasil, as regiões Nordeste, Centro-Oeste e Norte são as que apresentam maiores taxa de mortalidade, e os municípios pequenos, em função da falta de infraestrutura que dificulta a detecção e o tratamento da doença, apresentam menores taxas de mortalidade do que grandes centros urbanos ${ }^{17}$.

Conhecer o perfil epidemiológico de mulheres com neoplasia maligna da mama por meio de dados secundários é recomendado, uma vez que as limitações inerentes a um estudo descritivo são bem descritas na literatura.

Sabe-se que a qualidade das informações dos sistemas de informação depende diretamente do preenchimento correto, podendo não refletir com completude a situação investigada. Portanto uma das limitações observada no estudo foi o alto número de dados "sem informações", o que pode estar relacionado a um preenchimento inadequado das fichas de admissão dessas pacientes, instrumento que alimenta todo o sistema e traz números importantes, que devem ser precisos, para todo e qualquer plano de intervenção.

Outra limitação se refere ao fato de que os resultados não correspondem ao quadro real das internações por neoplasia maligna da mama em mulheres no estado do Bahia, pois foram analisados apenas os casos registrados pelo Sistema de Informação Hospitalar SIH (SUS). Em contrapartida, apenas informações do SUS são fundamentais para planejar programas, ações e políticas públicas com vistas a reverter o numero de internações e mortalidade de mulheres acometidas por neoplasia de mama. As internações no sistema privado de saúde estão excluídas desta análise.

\section{Conclusão}

Este trabalho permitiu identificar algumas características importantes das mulheres internadas com neoplasia maligna da mama na Bahia no período de 2012 a 2016. Foi observada uma tendência crescente quanto ao número de internações entre os anos estudados. A maior proporção de internação ocorreu nas mulheres com idade entre 50 a 59 anos e nas que se autodeclaram cor/ raça parda. A macrorregião do Leste do Estado, onde está localizada a capital Salvador, apresentou maior frequência de internações. Taxa de mortalidade entre as mulheres internadas foi maior na faixa etária de 50 a 59 anos de idade.

Os dados apresentados permitem traçar um perfil da morbidade e mortalidade por câncer de mama na Bahia e suas macrorregiões, marcadamente influenciada por desigualdades sociais e de acesso a serviços de saúde, e servir de subsídios para estratégias de enfrentamento do problema. Porém, é necessário um maior esforço dos profissionais e gestores no sentido da completitude das informações, especialmente as que representam importantes indicadores de saúde, a fim de que os levantamentos que utilizam dados secundários sejam mais fidedignos a realidade.

\section{Contribuições dos autores}

Matos BES, Rocha FC e Pereira NAM participaram da coleta de dados, análise e interpretação dos resultados e escrita do artigo. Cardoso ACC e Brasil CA participaram da análise e interpretação de dados, orientação e supervisão do artigo. Palmeia CS participou da redação do manuscrito e revisão da versão final.

\section{Conflitos de interesses}

Nenhum conflito financeiro, legal ou político envolvendo terceiros (governo, empresas e fundações privadas, etc.) foi declarado para nenhum aspecto do trabalho submetido (incluindo, mas não se limitando a subvenções e financiamentos, participação em conselho consultivo, desenho de estudo, preparação de manuscrito, análise estatística, etc.). 


\section{Referência}

1. Bray F, Ferlay J, Soerjomataram I, Siegel RL, Torre LA, Jemal A. Global Cancer Statistics 2018: GLOBOCAN Estimates of Incidence and Mortality Worldwide for 36 Cancers in 185 Countries. CA: a Cancer Journal for Clinicians, 2018;68(6):394-424. doi: 10.3322/ caac. 21492

2. Instituto Nacional de Câncer José Alencar Gomes da Silva. A situação do câncer de mama no Brasil: síntese de dados dos sistemas de informação. Rio de Janeiro: Instituto Nacional de Câncer José Alencar Gomes da Silva; 2019.

3. Allemani C, Matsuda T, Di Carlo V, Harewood R, Matz M, Nikšić $M$ et al. Global surveillance of trends in cancer survival 2000-14 (CONCORD-3): analysis of individual records for 37513025 patients diagnosed with one of 18 cancers from 322 populationbased registries in 71 countries. Lancet. 2018;391(10125):10231075. doi: $10.1016 / 50140-6736(17) 33326-3$

4. Siqueira ASE, Gonçalves JG, Balaro ML, Mendonça PEX, Merhy EE, Land MGP. Impacto econômico das internações, quimioterapias e afastamentos por Neoplasia Maligna de Mama no Brasil. DIVERSITATES International Journal. 2016;8(1).

5. Ministério da Saúde. Secretaria de Atenção à Saúde. Instituto Nacional do Câncer (INCA). Incidência do câncer no Brasil: estimativa 2008. [Internet]. 2008. [acesso em 2018 out. 20]. Disponível em: http://www.inca.gov.br/estimativa/2008/index. asp?link=conteudo_view.asp\&ID=2

6. Migowski A, Silva GA, Dias MBK, Diz MDPE, Sant'Ana DR, Nadanovsky P. Diretrizes para detecção precoce do câncer de mama no Brasil. II - Novas recomendações nacionais, principais evidências e controvérsias. Cad Saúde Pública. 2018;34(6):1-16. doi: 10.1590/0102-311×00074817

7. Tomazelli JG, Silva GA. Rastreamento do câncer de mama no Brasil: uma avaliação da oferta e utilização da rede assistencial do Sistema Único de Saúde no período 2010-2012. Epidemiol Serv Saúde. 2017;26(4):713-724. doi: 10.5123/s167949742017000400004

8. Frazão A, Skaba MMF. Mulheres com Câncer de Mama: as Expressões da Questão Social durante o Tratamento de Quimioterapia Neoadjuvante. Rev Brasileia de Cancerologia. 2013;59(3):427-435.

9. Guerra MR, Silva GA, Nogueira MC, Leite ICG, Oliveira RVC, Cintra JRD et al. Breast cancer survival and health iniquities. Cad Saúde Pública. 2015;31(8):1673-1684. doi: 10.1590/0102$\underline{311 \times 00145214}$

10. Medeiros GC, Bergmann A, Aguiar SS, Thuler LCS. Análise dos determinantes que influenciam o tempo para o início do tratamento de mulheres com câncer de mama no Brasil. Cad Saúde Pública. 2015;31(6):1269-1282. doi: 10.1590/0102$\underline{311 \times 00048514}$
11. Silva GA, Gamarra CJ, Girianelli VR, Valente JG. Tendência da mortalidade por câncer nas capitais e interior do Brasil entre 1980 e 2006. Rev Saúde Pública. 2011;45(6):1009-1018. doi: 10.1590/ $\underline{\mathrm{S} 0034-89102011005000076}$

12. Renna Junior NL, Silva GA. Late-Stage Diagnosis of Breast Cancer in Brazil: Analysis of Data from Hospital-Based Cancer Registries (2000-2012). Rev Bras Ginecol Obstet. 2018;40(3):127136. doi: $10.1055 / \mathrm{s}-0038-1624580$

13. Soares PBM, Quirino Filho S, Souza WP, Gonçalves RCR, Martelli DRB, Silveira MF et al. Características das mulheres com câncer de mama assistidas em serviços de referência do Norte de Minas Gerais. Rev Bras Epidemiol. 2012;15(3):595-604. doi: 10.1590/S1415-790X2012000300013

14. Ministério da Saúde. Portaria n 876, de 16 de maio de 2011. Dispõe sobre a aplicação da Lei no 12.732, de 22 de novembro de 2012, que versa a respeito do primeiro tratamento do paciente com neoplasia maligna comprovada no âmbito do Sistema Único de Saúde (SUS). Diário Oficial da União. 2013 mai.

15. Ho GF, Taib NA, Singh RKP, Yip CH, Abdullah MM, Lima TO. What If All Patients with Breast Cancer in Malaysia Have Access to the Best Available Care: How Many Deaths Are Avoidable?. Global Journal of Health Science. 2017;9(8). doi: 10.5539/gjhs.v9n8p32

16. Souza MM, Winnikow EP, Moretti GP, Panatto APR, Rosa MI, Simões PWTA. Taxa de mortalidade por neoplasia maligna de mama em mulheres residentes da Região Carbonífera Catarinense no período de 1980 a 2009. Cad Saúde coletiva. 2013;21(4):384-90. doi: 10.1590/S1414-462X2013000400005

17. Couto MSA, Guerra MR, Firme VAC, Bustamante-Teixeira MT. Comportamento da mortalidade por câncer de mama nos municípios brasileiros e fatores associados. Rev Panam Salud Publica. 2017;41:e168. doi: 10.26633/RPSP.2017.168 